\title{
PHONON TRANSPORT AT THE NANOSCALE WITH APPLICATIONS TO BATTERIES AND ADVANCED THERMAL INSULATION
}

\author{
Sean Lubner ${ }^{1}$, Sumanjeet Kaur ${ }^{1}$, Joseph Franklin ${ }^{1,2}$, Ravi Prasher ${ }^{1,3 *}$ \\ ${ }^{1}$ Lawrence Berkeley National Lab, Berkley, California, USA \\ ${ }^{2}$ Electrochemical Innovation Lab, Department of Chemical Engineering, University College London, \\ London, WC1E 7JE, UK \\ ${ }^{3}$ University of California, Berkeley, California, USA
}

\begin{abstract}
It has been almost three decades since Nanoscale Thermal Science and Engineering became a wellestablished research field. Various major breakthroughs in fundamental understanding of thermal transport (phonons, photons, and electrons) at the nanoscale have been achieved in these three decades; however, the impact of these fundamental insights has been primarily targeted toward microelectronics and thermoelectrics applications. In this paper we provide examples of other applications such as Lithium ion battery thermal management and building thermal insulation, where nanoscale thermal science has a significant role to play. We have used time domain thermoreflectance (TDTR) to measure thermal conductivity of Lithium ion battery cathode material. To understand the fundamentals of thermal transport in the cathode material we created a model cathode system as compared engineered samples using pulsed laser deposition technique. We also used 3-omega technique for the engineered system. We have also made highly insulating material using functionalized nanoparticles for building applications. Results show that surface functionalization has a huge impact on thermal conductivity of an assembly of nanoparticle.
\end{abstract}

KEY WORDS: Battery thermal management, Building thermal insulation

\section{INTRODUCTION}

In this paper two examples of nanoscale thermal science are discussed from the field of energy: 1) Electrochemical Batteries: Batteries are going to play a significant role in the future for both mobile and stationary applications. Thermal management is very important for batteries. 2) Building thermal insulation: Cooling and heating of buildings consumes $15 \%$ of the primary energy in the U.S. For increased energy efficiency of buildings it is very important to develop the next-generation of insulations that are cost effective and retroactively compatible with existing infrastructure.

\section{NANO-SCALE BATTERY THERMAL TRANSPORT MEASUREMENTS}

\subsection{Introduction and Background}

Heat dissipation efficiency within batteries directly affects their performance, reliability, safety, and maximum recharge/discharge rates [1], [2]. Enhancing the internal cross-plane thermal conductivity $(k)$ within batteries would enable a significant reduction in the need for bulky and expensive external forced convection cooling systems [3], improving effective battery power density and energy density. It would reduce internal temperature gradients, alleviating thermal stresses and degradation due to battery cycling and 
ambient temperature oscillations. Finally, it would lower the peak internal battery temperature rise, allowing for enhanced safety and increased maximum recharge and discharge rates. A recent preliminary experimental study [4] on Li-Ion battery cells implied that the thermal interface resistance at the cathodeseparator interface is the dominant factor leading to low overall effective $k$ inside batteries and their poor thermal performance. The findings suggest that eliminating this thermal resistance would improve the effective $k$ by more than 8 times.

To better investigate this problem we are utilizing two state of the art thermal metrologies to measure nanoscale and interfacial thermal transport within batteries. We are using a modified multilayer 3-omega technique [5]-[7] to fabricate sensors within live battery pouch cells to measure the thermal transport across each layer and interface during operation. 3-Omega is a frequency domain electrothermal technique that launches dissipative thermal waves into samples to measure their thermal transport properties. However, the 3 -omega technique can only provide mesoscopic measurements with several-microns scale resolution at best. To provide a complete solution to the thermal bottleneck a full understanding of the thermal transport physics at the cathode layer interface is necessary requiring nanometer-scale thermal measurements. We are therefore also using time domain thermoreflectance (TDTR) [8], [9] to measure the intrinsic thermal transport properties of carefully prepared model systems of the active material in Li-ion cathodes. TDTR is an ultrafast pump-probe laser technique capable of measuring thermal transport properties at the nano-scale. By combining both of these approaches we hope to provide enough data to allow for the development of an accurate all-scale hierarchical thermal model of heat transport within batteries. In this paper we will focus primarily on the nano-scale TDTR measurements and only briefly mention our progress being made with 3omega.

\subsection{Experimental Approach}

In order to perform nano-scale optical measurements, an ideal model experimental system is needed. The real battery system is an engineered composite system comprising additives and binders where crystal orientation and the grain size of the active battery material cannot be well controlled. Therefore we created epitaxial nm-scale active battery cathode materials for study with TDTR (Fig. 1). These ideal systems have well defined low-roughness surfaces $(<1 \mathrm{~nm} \mathrm{rms})$ with controllable single crystal orientations, and allow us to measure the thermal conductivity of the isolated active material without obfuscation from the other aspects of the messy and complicated composite material of an actual battery cathode.
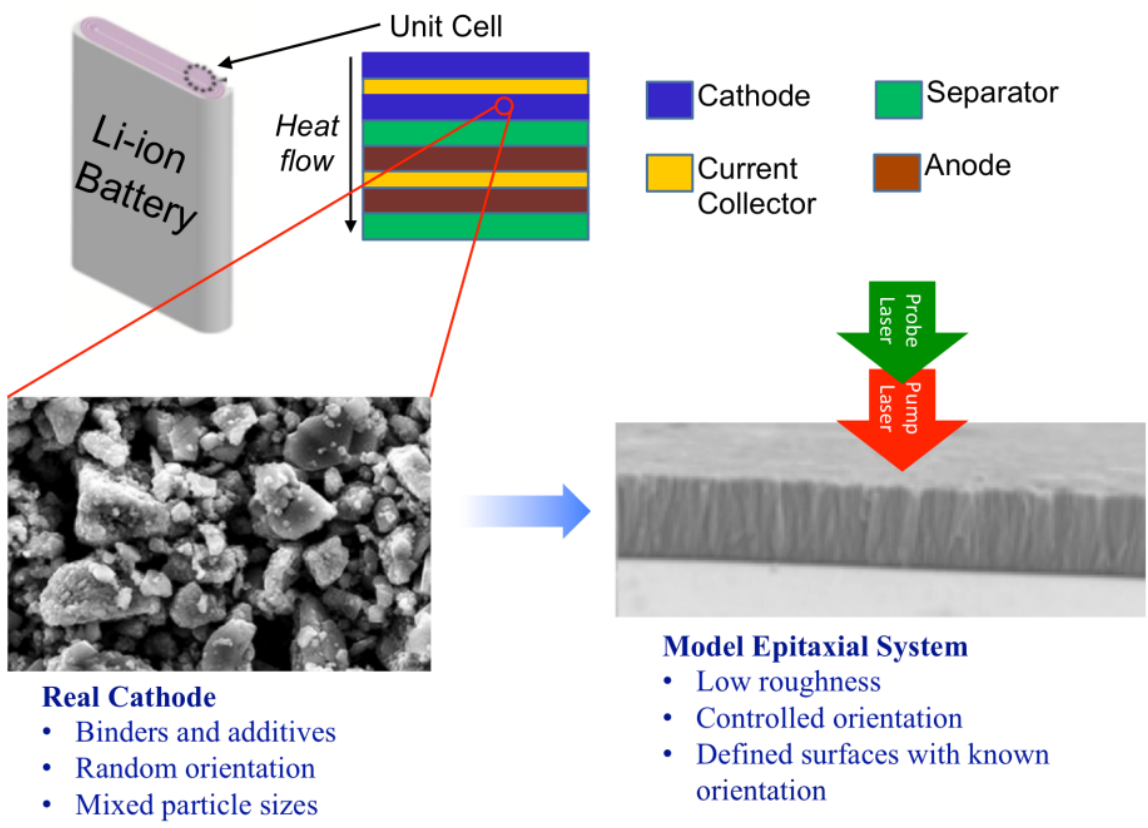

Model Epitaxial System

- Low roughness

- Controlled orientation

- Defined surfaces with known orientation 
Fig. 1. (Top) Heat must flow across all layers of the repeating unit cell to escape the lithium-ion battery. (Bottom left) SEM image of a real cathode; too messy to get a controlled thermal transport measurement of the isolated active material. (Bottom right) SEM image of epitaxial grown pristine cathode material ideal for TDTR cross-plane thermal conductivity measurements.

To create these model systems, we use pulsed laser deposition to grow $50 \mathrm{~nm}$ thick epitaxial $\mathrm{LiNi}_{0.5} \mathrm{Mn}_{1.5} \mathrm{O}_{4}$ (LNMO) pristine cathode material on the 0001 (C-plane) and 11(-2)0 (A-plane) faces of sapphire substrates (Fig. 2). The different substrate orientations resulted in different crystal orientations of the deposited LNMO film, enabling us to measure the anisotropic thermal conductivity tensor of the cathode active material. Before measurement, samples were coated in $70 \mathrm{~nm}$ of $\mathrm{Al}$ as an optical transducer layer necessary for TDTR.

(a)

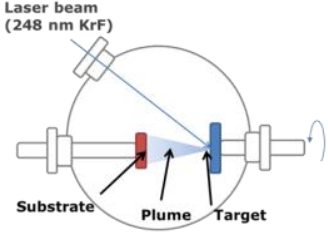

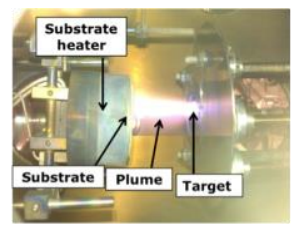

(b)

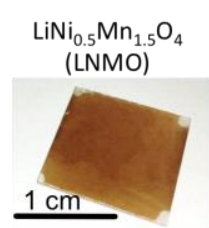

(c)

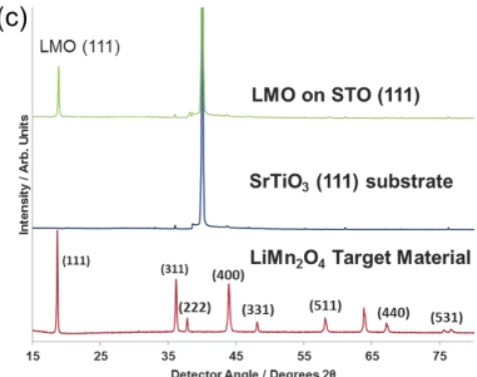

Fig. 2. Creation of pristine model battery system for thermal study. (a) Pulsed laser deposition system with recipes optimized for our sample. (b) Example of an as-fabricated LNMO cathode sample. (c) X-ray diffraction data showing atomic structure of fabricated samples including their substrates.

\subsection{Results and Discussion}

An example of the raw fitted TDTR data is shown in Fig. 3. The two different sample crystal orientations allowed us to measure the LNMO thermal conductivity, $k$, in both principle directions. We measured $k_{0001}=1.4 \mathrm{~W} / \mathrm{m}-\mathrm{K}$ and $k_{11(-2) 0}=3.8 \mathrm{~W} / \mathrm{m}-\mathrm{K}$, yielding an anisotropy ratio of 2.7. This information helps us understand the thermal transport through battery cathodes from the nano-scale up. Models of battery cathode thermal transport must account for distributions of grains with randomly oriented anisotropic thermal conductivity tensors. These results further our ability to predict changes to the bulk effective $k$ of the system-level cathode composite as a result of changes to the active material. These nano-scale measurements will be combined with future 3-omega micro-scale thermal measurements for a complete thermal transport picture of the battery. 

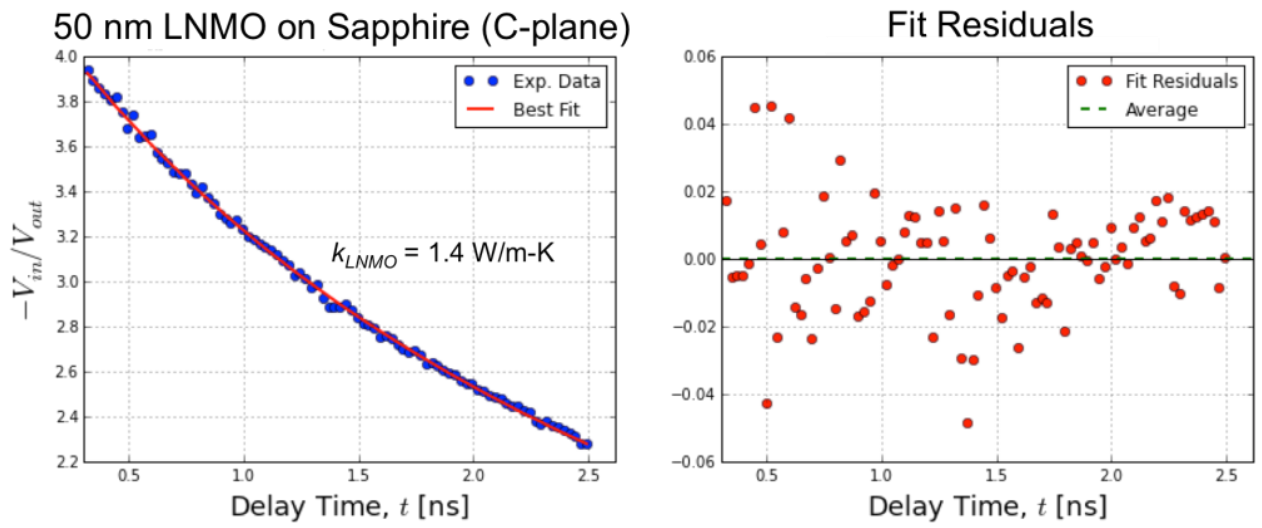

Fig. 3. Representative raw TDTR data for one of the LNMO thermal conductivity measurements. The randomly distributed fit residuals centered on 0 indicate a good fit to the data.

In addition to these TDTR measurements, we are also using embedded 3-omega sensors to measure thermal interfacial resistance between battery layers in-situ. We intend to publish the full details of these measurements soon. Our preliminary results confirm the cathode-separator interface as being the primary thermal bottleneck for heat dissipation in batteries, with a very low in-situ thermal boundary conductance of $962 \mathrm{~W} / \mathrm{m}^{2}-\mathrm{K}$ (with electrolyte present) constituting $92 \%$ of the total thermal resistance through the battery unit cell.

To summarize this section, we have used pulsed laser deposition to create TDTR-compatible model battery cathode systems. By growing LNMO on two substrates with different crystal orientations we were able to control the orientation of the grown film itself. This in turn enabled us to measure the cross-plane thermal conductivity in both directions using TDTR, yielding both direction's value of $k$ and the anisotropy ratio. Using a modified 3-omega technique our preliminary results indicate that the cathode-separator interface is the primary thermal bottleneck. These results begin to pave the way toward a complete understanding of thermal transport within batteries. Our measurements motivate how being able to enhance phonon transport across targeted interfaces and understand heat transport from the nano-scale up could lead to significant improvements to thermal management in battery technology. In the next section, we will show how applying these same principles in reverse to use a nano-scale understanding of heat transport to instead reduce phonon transport across interfaces can lead to significant improvements for thermal insulation technology.

\section{NANOPATICLE BASED THERMAL INSULATION FOR BUILDINGS}

\subsection{Introduction and Background}

The buildings sector accounts for 41 percent ( 40 quads) of The United States' energy consumption-more than any other end-use sector. Space heating and cooling represents $\sim 36$ percent (14.5 quads) of that energy used in buildings. High-performance and cost-effective thermal insulation can significantly reduce cooling and heating loads. The U.S. buildings sector is currently dominated by buildings already in existence. Therefore, a significant market for building insulation is the retrofit market. Size and design constraints make it very challenging to retrofit existing buildings with conventional insulations. Retrofit projects require using an insulation that, apart from meeting other required properties, has a high $\mathrm{R}$-value $\left(\mathrm{h} \cdot \mathrm{ft}^{2} \cdot{ }^{\circ} \mathrm{F} / \mathrm{Btu}\right) \mathrm{per}$ inch, and is cost-effective and easy to install.

The most commonly used building insulation materials are fiberglass, rock wool, expanded polystyrene (EPS), extruded polystyrene (XPS), polyisocyanurate and cellulose. Although conventional insulation materials are relatively inexpensive, they are not feasible candidates for retrofits. Because of their low R per inch $(3-6)$, conventional thermal insulation materials typically take the form of mats or boards that are 2 to 
4 inches thick to provide for R12 insulation. Such thick insulations are not practical because they reduce living space when placed on interior walls or require significant alteration of window/door openings and face zoning regulations when used externally on walls. They also make it significantly more difficult for interior installations such as shelves, cabinets, and plumbing \& electrical wiring constraints to access the structurally robust portion of the building walls. Other emerging insulation solutions, such as aerogel and vacuuminsulated panels (VIPs) have high R/inch values but at a much higher cost. Aerogel, the world's lightest solid material, typically contains 95 percent to 99 percent air by volume. It is made using a sol-gel process whereby a highly porous solid network is achieved by replacing the liquid inside the material's pores with gas. The process requires super critical drying [10]. Super critical drying is used to avoid the creation of capillary forces, which would collapse the highly porous and fragile solid network during conventional evaporation of the liquid phase. This integral step is also the primary barrier to commercialization of aerogel, because it requires high temperatures and/or high pressures that consume large amounts of energy. The highenergy requirements and the batch processing of aerogel limit the scaling up of aerogel manufacturing, which continues to make it very expensive. In addition, aerogel's low volume fraction of solids (1 percent to 5 percent) makes it extremely fragile and difficult to handle (Fig. 4a) unless it is integrated or reinforced with a fibrous matrix. Silica aerogel in its pristine form can have a thermal conductivity as low as $0.01 \mathrm{~W} / \mathrm{m}$ $\mathrm{K}$, but when integrated with a fibrous matrix for practical applications, the thermal conductivity of the final product increases to $0.014 \mathrm{~W} / \mathrm{m}-\mathrm{K}$ (www.aerogel.com).

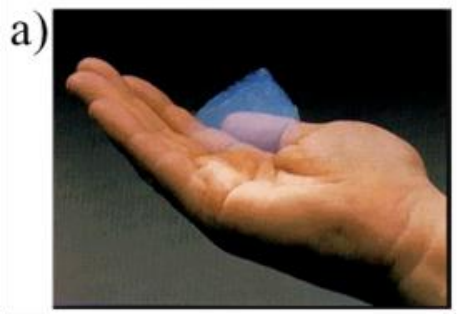

b)

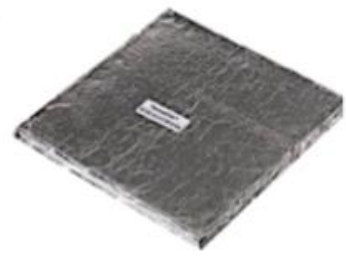

Fig. 4. a) Monolithic silica aerogel, b) vacuum insulated panel (VIP)

VIPs consist of an open-pore core material that is vacuum-enveloped by foil that prevents the entry of air and water vapor as shown in Fig. 4b. Since the R/inch values of VIPs is between R35 - R45, a very thin (9 mm) VIP can provide thermal insulation equivalent to thick mats $(76$ to $100 \mathrm{~mm})$ of conventional insulation materials. VIPs, however, suffer from several limitations [10]. Because they are vacuum-enveloped, VIPs come in precut forms and cannot be cut or modified at the building site, making them generally unsuitable for retrofits, which typically require a custom fit. VIPs are also vulnerable to being punctured by nails or screws, which could decrease their R/inch value to around 7. In addition, the performance of VIPs tends to decline over time because the foil is not completely impermeable to air and moisture.

There is no insulation on the market that can cost-effectively retrofit a building to make its building envelope energy efficient. There is a clear need to develop a robust insulation conducive to retrofit projects that has a high R-value and can be sold at a competitive price. So far the R\&D efforts in this field have centered on achieving high $\mathrm{R}$-values by either reducing the solid volume fraction (as in aerogel) or making vacuumenclosed panels.

In this work we propose to achieve very low thermal conductivity cost-effectively by manipulating the heat transfer at the nano scale. Interfaces between materials create discontinuity in the heat flow due to the scattering of phonons (fundamental heat carriers in non-metals) at the interfaces. In the case of nanomaterials, which have a very high density of interfaces, it is therefore possible to attain super low thermal conductivities at high solid volume fraction making the insulation mechanically robust. We therefore used the approach of combining nanoparticles into bulk materials to maximize interface densities and phonon scattering, minimizing thermal conductivity. Manufacturing and scale-up of nanoparticle-based insulation does not suffer from the limitations of aerogels due to the use of very low energy requirements, thereby making the anticipated cost of our material significantly cheaper than aerogels. Our proposed 
insulation although mechanically robust, will be much more flexible and modular than VIP so that it can be bent and cut on-site into any shape or size required, thanks to the use of randomly sprayed nanoparticles on a flexible substrate.

Theoretically, it was shown by Prasher that the effective mean free path (m.f.p) of phonons ( $\left.l_{\text {eff }}\right)$ for two nanoparticles in contact with each other (Fig. 5a) is given by [11],

$$
l_{\text {eff }}=3 \frac{a^{2}}{R} \quad \sin () \cos () d
$$

where $a$ is the constriction radius (Fig. 5a), $R$ is the radius of the nanoparticle and $\tau$ is the transmissivity of the phonons. Thermal conductivity, $k$, is given by $(1 / 3) c v l$ where $c$ is the phonon specific heat per unit volume, $v$ is the Debye speed (group velocity of phonons) and $l$ is the m.f.p. $a^{2} / R$ in Eq. (1) is given by $(1.125 \pi \gamma / E)^{2 / 3} R^{1 / 3}$ where $\gamma$ is the surface energy and $E$ is the effective modulus. $a^{2} / R$ can be very small for nanoparticles depending on $\gamma, E$ and $R$. The phonon transmissivity, $\tau$ in Eq. (1), is a function of $\gamma$ and acoustic properties of the nanoparticles [12]. For smaller values of $\gamma, \tau$ can be very small. Eq. (1) shows that the primary knobs to get very low thermal conductivity are 1) surface energy 2) nanoparticle size and 3) acoustic property mismatch by mixing different types of particles. It is therefore plausible to have two scenarios as shown in Fig. 5b that will enable the nanoparticle bed to have lower thermal conductivity than aerogel. In one scenario $\gamma$ is reduced and in the other acoustic mismatch is generated by using two different materials (Silica and Alumina for example). This provides the conceptual underpinning of our approach to create an ultra-low thermal conductivity material out of nanoparticles. Both these scenarios show that it's possible to beat aerogel for nanoparticle diameter in the range of 50 to $100 \mathrm{~nm}$. These scenarios and the experimental data collected by the PI and co-workers [13] on packed bed of randomly distributed alumina nanoparticles (volume fraction $\sim 0.6$, are shown in Fig. 5c. $k$ of bulk alumina is $\sim 25 \mathrm{~W} / \mathrm{m}-\mathrm{K}$. The experimental data (black dots) in Fig. 5c shows that $k$ of the packed nanoparticle bed of alumina nanoparticles is $\sim 3$ orders of magnitude less and is close to that of air. This experimental data is compared with the theoretical model given by Eq. (1) and it shows a very good match between data and the model, which enables us to have good confidence in the model. In fact [14] used the idea of mismatch in acoustic properties by mixing different nanoparticles (Alumina, Silica and carbon black) and got thermal conductivity as low as $0.01 \mathrm{Wm}-\mathrm{K}$ (same as aerogel) at a particle volume fraction of $50 \%$ which is consistent with the results shown by the model in Fig. 5c providing further confidence in the model and the underlying physics. In fact based on our model, combination of lower surface energy and acoustic mismatch leads $\mathrm{k}$ as low as $0.005 \mathrm{~W} / \mathrm{m}-\mathrm{K}(\sim 50 \%$ of aerogel $)$. 

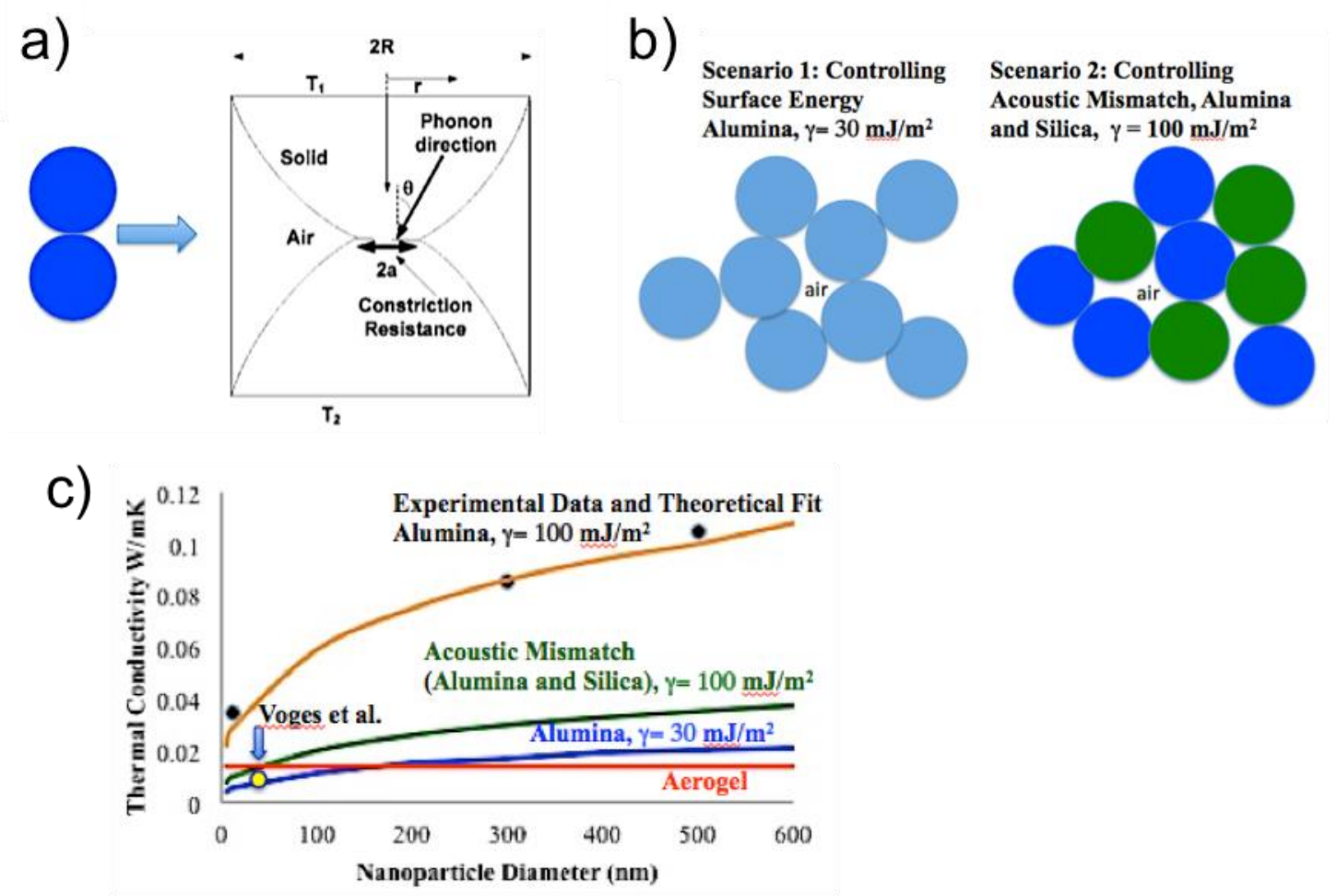

Fig. 5. a) Schematic showing nanoparticle and constriction; b) schematic showing randomly packed nanoparticles; c) The black dots are the experimental data from [13] showing the effect of size of alumina nanoparticles on $k$; solids lines represents the effect of various parameters on $k$ as predicted by the theoretical model; the yellow dot represents $k$ of nanoparticle mixture from [14].

\subsection{Experimental Approach}

We used the Hot Disk metrology for thermal measurements of our samples [15], [16]. The Hot Disk technique is a well established method with particularly good sensitivity for thermal conductivity, including for highly insulating samples, and these details can be found in refs [15]-[17]. Briefly, the Hot Disk technique, also known as the transient plane source (TPS) technique, is a transient thermal measurement that calculates thermal conductivity and volumetric heat capacity $\left(C_{v}\right)$ from a measured transient heating curve. For our measurements, we used the commercially available "Hot Disk TPS 500 S" model from ThermTest Inc. For these measurements, we embedded the Hot Disk sensor within a cylindrical volume of our nanoparticle bed sample approximately $2 \mathrm{~cm}$ in diameter $\mathrm{x} 2 \mathrm{~cm}$ thick. Care was taken to ensure the thermal penetration of the heating from the sensor never reached any sample boundaries.

\subsection{Results and Discussion}

We investigated the effects of nanoparticle size and acoustic mismatch between nanoparticles on the thermal properties nanoparticle beds. Our results are discussed herein.

\subsubsection{Size effect}

We investigated size effect for silica and titania nanoparticles. We see a decrease in thermal conductivity with size. This is consistent with the theory, as reducing size reduces the constriction radius, $a$, and increases the interface density and hence rate of phonon scattering. Preliminary data is shown in Fig. 6. 

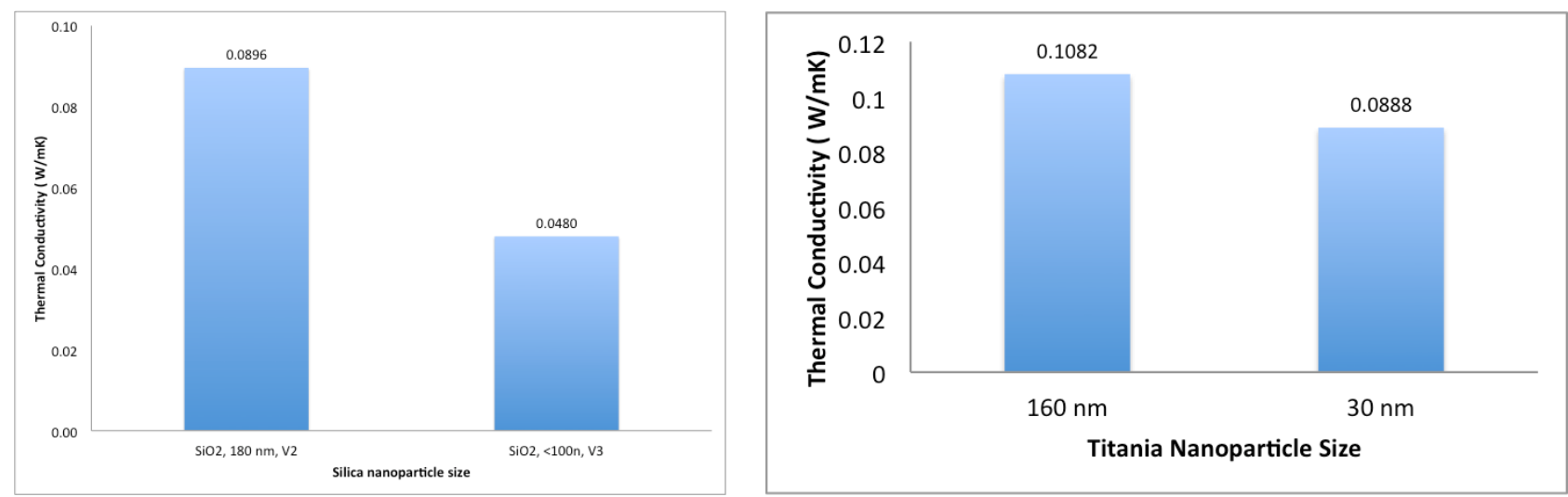

Fig. 6. Effect of particle size on thermal conductivity.

\subsubsection{Acoustic Mismatch}

Two sets of samples were studied for the effect of acoustic mismatch. Set 1 consists of $\mathrm{SiO}_{2}$ and $\mathrm{TiO}_{2}$ in a 2.3:1 $\left(\mathrm{SiO}_{2}: \mathrm{TiO}_{2}\right)$ volumetric ratio. Set 2 consists of $\mathrm{SiO}_{2}$ and $\mathrm{Al}_{2} \mathrm{O}_{3}$ in a $1: 1\left(\mathrm{SiO}_{2}: \mathrm{Al}_{2} \mathrm{O}_{3}\right)$ volumetric ratio. The measured thermal conductivities from these samples are shown in Fig. 7. Initial data shows the trend of decreasing thermal conductivity for the mixed samples (acoustic mismatch samples).
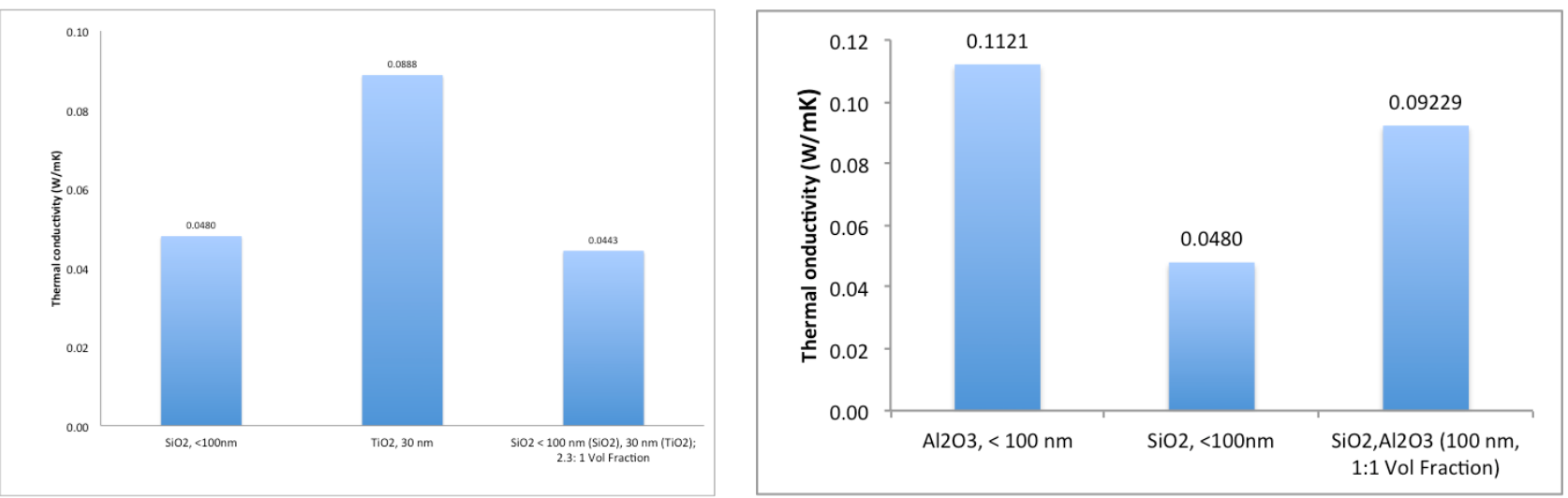

Fig. 7. Effect of acoustic mismatch on thermal conductivity for Set 1 and Set 2 samples.

To summarize this section, we applied multiple theoretical results to engineer a low thermal conductivity material suitable for building insulation retrofit installations. Our material is made from packed nanoparticle beds. We use small nanoparticles constituting a mixture of different materials. The small size leads to small constriction radii between particles and high interface density of the macroscopic solid, maximizing the scattering of phonons and hence reducing thermal conductivity. The mixture of materials leads to a high acoustic mismatch among adjacent dissimilar particles, owing to their different phonon density of states. This further reduces the ability for phonons to transmit among nanoparticles, further reducing the bulk thermal conductivity. Our nanoparticle-based material can be cheaply manufactured at scale, and can be bent and cut to different shapes and sizes on-site without losing its thermal properties. This combination of properties makes it an ideal material for building insulation retrofit applications.

\section{CONCLUSIONS}

We have demonstrated two areas where micro and nanoscale heat transfer concepts can be applied to solve major real world problems. We created sensors that were able to measure in-situ the thermal transport across microscopic layer interfaces within a functioning battery cell with liquid electrolyte. These measurements revealed that the primary thermal bottleneck within batteries resides at the interface between the cathode and separator. Better modeling and ultimately enhancing the thermal transport at this interface and throughout the batteries' internal multilayer stacks will lead to better battery performance, safety, and reliability, and will help to enable the extreme fast charging of batteries necessary for future electric vehicles. We then applied the fundamentals of phonon transport and scattering to design and create a cheap, flexible, and robust nanoparticle-based thermal insulation material ideal for retrofitting energy inefficient buildings, leading to 
potentially huge energy savings nationwide. These two projects demonstrate the wealth of unrealized utility in applying the principles of micro and nano scale thermal transport physics to real devices and problems.

\section{ACKNOWLEDGMENT}

Building thermal insulation work was supported by the Assistant Secretary for Energy Efficiency and Renewable Energy, Building Technologies Program, of the U.S. Department of Energy under Contract No. DEAC02-05CH11231. Battery thermal work was support by the Assistant Secretary for Energy Efficiency and Renewable Energy, Vehicle Technologies Office, under the Advanced Battery Materials Research (BMR) Program, of the U.S. Department of Energy under Contract No. DE-AC02-05CH11231. JBF acknowledges support from the European Union Horizon 2020 under the Marie Sklodowska-Curie grant agreement No. 705339 and is grateful for support from the Science and Technology Facilities Council Early Career award, ST/K00171X/1.

\section{REFERENCES}

[1] M. R. Palacin and A. de Guibert, "Why do batteries fail?," Science (80-. )., vol. 351, no. 6273, pp. 1253292-1253292, 2016.

[2] T. M. Bandhauer, S. Garimella, and T. F. Fuller, "A Critical Review of Thermal Issues in Lithium-Ion Batteries," J. Electrochem. Soc., vol. 158, no. 3, p. R1, 2011.

[3] D. Chen, J. Jiang, G. H. Kim, C. Yang, and A. Pesaran, "Comparison of different cooling methods for lithium ion battery cells," Appl. Therm. Eng., vol. 94, pp. 846-854, 2016.

[4] V. Vishwakarma, C. Waghela, Z. Wei, R. Prasher, S. C. Nagpure, J. Li, F. Liu, C. Daniel, and A. Jain, "Heat transfer enhancement in a lithium-ion cell through improved material-level thermal transport," J. Power Sources, vol. 300, pp. 123131,2015

[5] D. G. Cahill, "Thermal conductivity measurement from 30 to $750 \mathrm{~K}$ : the $3 \omega$ method," Rev. Sci. Instrum., vol. 61, no. 2, pp. 802-808, 1990.

[6] C. Dames and G. Chen, " $1 \omega, 2 \omega$, and $3 \omega$ Methods for Measurements of Thermal Properties," Rev. Sci. Instrum., vol. 76, no. 12, p. 124902, 2005.

[7] A. Feldman, "Algorithm for solutions of the thermal diffusion equation in a stratified medium with a modulated heating source," High Temp. Press., vol. 31, no. 3, pp. 293-298, 1999.

[8] D. G. Cahill, "Analysis of heat flow in layered structures for time-domain thermoreflectance," Rev. Sci. Instrum., vol. 75, no. 12, pp. 5119-5122, Dec. 2004.

[9] A. J. Schmidt, X. Chen, and G. Chen, "Pulse accumulation, radial heat conduction, and anisotropic thermal conductivity in pump-probe transient thermoreflectance," Rev. Sci. Instrum., vol. 79, no. 11, pp. 1-9, 2008.

[10] R. Baetens, B. P. Jelle, and A. Gustavsen, “Aerogel Insulation for Building Applications: A state-of-the-art review,” Energy Build., vol. 43, pp. 1385-1412, 2011.

[11] R. Prasher, "Ultralow thermal conductivity of a packed bed of crystalline nanoparticles: A theoretical study," Phys. Rev. B Condens. Matter Mater. Phys., vol. 74, no. 16, pp. 1-5, 2006.

[12] R. Prasher, "Acoustic mismatch model for thermal contact resistance of van der Waals contacts," Appl. Phys. Lett., vol. 94, no. 4, pp. 2007-2010, 2009.

[13] X. J. Hu, R. Prasher, and K. Lofgreen, "Ultralow thermal conductivity of nanoparticle packed bed," Appl. Phys. Lett., vol. 91, no. 20, pp. 11-13, 2007.

[14] K. Voges, M. Vadala, and D. C. Lupascu, "Dense nanopowder composites for thermal insulation," Phys. Status Solidi Appl. Mater. Sci., vol. 212, no. 2, pp. 439-442, 2015.

[15] S. E. Gustafsson, "Transient diffusivity plane source techniques for thermal conductivity measurements of solid materials and thermal," Rev. Sci. Instrum., vol. 62, no. March, pp. 797-804, 1991.

[16] Y. He, "Rapid thermal conductivity measurement with a hot disk sensor: Part 1 . Theoretical considerations," Thermochim. Acta, vol. 436, no. 1-2, pp. 122-129, 2005.

[17] S. Malinarič, "Uncertainty analysis of thermophysical property measurements of solids using dynamic methods," Int. $J$. Thermophys., vol. 28 , no. 1 , pp. 20-32, 2007. 